\title{
Minimally Invasive Pelvic Reconstructive Surgery: A Literature Review of Laparoscopic Surgery for Pelvic Organ Prolapse
}

\author{
Michelle M. Takase-Sanchez • Douglass S. Hale
}

Published online: 3 July 2013

(C) Springer Science+Business Media New York 2013

\begin{abstract}
Pelvic reconstructive surgery for pelvic organ prolapse includes transvaginal, open, laparoscopic, and roboticassisted approaches. Laparoscopy has established a significant role in minimally invasive surgery across surgical disciplines. In pelvic surgery, although the vaginal approach may offer the most native route to a minimally invasive technique, advances in gynecologic laparoscopy have reported advantages over traditional routes maintaining safety, efficacy, and high patient satisfaction. Majority of current data is limited to descriptive case series and retrospective data that nonetheless continue to support the laparoscopic approach as a reasonably safe alternative to open and vaginal approaches. Few prospective, clinical trials have compared the safety, efficacy, and costeffectiveness of various approaches and surgical techniques highlighting challenges in the utility of robotic-assistance and vaginal graft placement. This literature review provides a summary of important historical and current data in regards to surgical technique and clinical outcomes of advanced pelvic laparoscopy for pelvic organ prolapse.
\end{abstract}

Keywords Pelvic organ prolapse · Pelvic reconstructive surgery $\cdot$ Minimally invasive gynecology $\cdot$ Urogynecology · Laparoscopy $\cdot$ Robotic-assisted · Anterior repair .

Paravaginal repair - Cystocele $\cdot$ Posterior repair $\cdot$ Rectocele $\cdot$ Enterocele $\cdot$ Hysteropexy $\cdot$ Cervicopexy $\cdot$ Uterosacral suspension $\cdot$ Colpopexy $\cdot$ Sacrocolpopexy $\cdot$ Sacral colpopexy $\cdot$ Mesh $\cdot$ Graft

\section{Introduction}

In recent decades, pelvic laparoscopy has progressed from a simple diagnostic tool to a complex armamentarium of advanced

M. M. Takase-Sanchez $(\varangle) \cdot$ D. S. Hale

1633 N. Capitol Ave, Suite 436, Indianapolis, IN 46202, USA

e-mail: mmtsanchez88@gmail.com surgical procedures. Advantages include superior visualization, magnification, reduced blood loss, decreased postoperative pain, decreased adhesion formation, and fewer wound complications $[1-3,4 \bullet \cdot, 5-7]$. Like any surgical procedure, increased volume is required to achieve these advantages after overcoming the initial learning curve [8]. Disadvantages of laparoscopy include costs of specialized equipment, disposable instruments, and the need for specialty-trained surgical staff, particularly in respects to robotic-assisted laparoscopy $[9 \bullet, 10 \bullet]$.

The objective of this chapter is to provide a review of current literature in regards to surgical technique and clinical outcomes of advanced laparoscopy as it applies to minimally invasive pelvic reconstructive surgery for pelvic organ prolapse. Although current literature lacks adequately powered, prospective, randomized control trials to provide conclusive outcomes analyses, the best available data that is pertinent to current clinical practice will be presented.

\section{Laparoscopic Surgical Procedures}

Anterior Compartment

Laparoscopic Paravaginal Cystocele Repair

G.R. White (1909) introduced the concept of a lateral anterior wall prolapse and described the paravaginal repair as a vaginal surgical approach to cystocele repairs by suturing the "lateral sulci to the white line of the pelvic fascia" [11]. Anatomic studies by Delancey et al. (2002) support this hypothesis that lateral defects are associated with cystocele development and bladder neck hypermobility [12]. Paravaginal repairs aim to correct lateral defects by reconnecting the fibromuscular vaginal tissue that has avulsed from its lateral attachment to the arcus tendineus fascia pelvis. Additional investigations on the pathophysiology of cystoceles revealed the complex interaction and strong correlation between apical 
and anterior support that is dynamic with varying degrees of levator ani impairment [13-15].

Literature on paravaginal repairs performed laparoscopically as an isolated procedure is limited. Behnia-Willison et al. (2007) reported 212 patients who underwent laparoscopic bilateral paravaginal repair with a $95 \%$ follow-up rate at a mean of 14.2 months. The objective anatomic cure rate (POP-Q $\leq$ Stage 1) was $76 \%$. Approximately $19.8 \%$ (42/212) had concurrent uterosacral ligament vault suspensions for concomitant Level I defects [16]. This data highlights the clinical significance of restoring apical Level I support when addressing anterior and paravaginal repairs [13-16]. A notable abdominal paravaginal study by Shippey et al. (2010) retrospectively compared outcomes on patients undergoing abdominal sacrocolpopexy with or without paravaginal repair (PVR) looking primarily at postoperative anterior failures defined as $\mathrm{POP}-\mathrm{Q}$ point $\mathrm{Ba} \geq-1 \mathrm{~cm}$. Results were limited by the observational design and a small sample size that was not powered to reach statistical significance; however, data suggested a trend toward reduced anterior failures when paravaginal repairs were performed concurrently with abdominal sacrocolpopexy [17•].

In summary, adequately powered studies designed specifically to evaluate the impact of paravaginal repairs on anatomic and clinical outcomes of prolapse surgery are needed. Furthermore, limited available data suggest that paravaginal repairs performed laparoscopically have at best similar anatomic success rates $(76 \%)$ compared with the open and vaginal paravaginal repair $(76-100 \%)[16,18]$.

\section{Middle Compartment: Apex}

Minimally invasive pelvic surgery to correct apical prolapse primarily includes laparoscopic hystero/sacrocolpopexy and the native tissue alternative of uterosacral ligament suspension. Total vaginal mesh and traditional vaginal colpopexy techniques are not reviewed here.

\section{Laparoscopic Uterosacral Colpopexy (Native Tissue Repair)}

The laparoscopic technique mimics the currently accepted "gold standard" transvaginal approach as a suspension of the vaginal apex as described by Shull et al. (2000). It involves the placement of sequentially higher sutures from each uterosacral ligament to the anterior and posterior vaginal fibromuscularis [19]. The pooled rate for apical success using the vaginal route is $98.3 \%$ (95\% confidence interval (CI) 95.7-100\%), supporting the durability of native tissue repairs done in this fashion [20•]. However, ureteral compromise was the most notable surgical risk unique to this procedure with a reported risk as high as $11 \%$ in one small series [21]. Therefore, expert opinion supports the universal use of intraoperative cystoscopy in these cases.
Limited data are available on laparoscopic uterosacral colpopexy. Several comparison studies of vaginal and laparoscopic techniques support the durability of the uterosacral colpopexy independent of surgical approach. Culligan et al. (2003) demonstrated that laparoscopically placed sutures have as much tensile strength as vaginally placed sutures [22]. Retrospective data from Rardin et al. (2009) suggests that the laparoscopic approach $(\mathrm{n}=22)$ may provide advantages over the vaginal approach $(\mathrm{n}=96)$ with a reduction of ureteral injuries from $4.2 \%$ to $0 \%(p=0.33)$. Although the outcomes of interest did not reach statistical significance, this study was one of the first to demonstrate trends toward lower recurrent symptomatic vault prolapse (10\% vs. $0 \%$ ), any symptomatic prolapse recurrence $(12.5 \%$ vs. $4.6 \%)$, and lower apical failure rates $(6.3 \%$ vs. $0 \%)$ with the laparoscopic approach (all $p$ values $>0.05$ ). Data that approached statistical significance included higher postoperative POPQ point $\mathrm{C}$ in the laparoscopic group $(-7.0$ vs. -5.9 , $p=0.04)$ and less postoperative vaginal cuff granulation tissue ( $15.8 \%$ vs. $0 \%, p=0.046)$ [23]. Advantages cited for the laparoscopic approach included ureteral visualization, ability to make a ureteral peritoneal relaxing incision, and lastly the ability to place additional sutures for more support if the initial sutures did not adequately elevate the cuff, an adjustment not possible using the vaginal approach. However, adequately powered comparison studies are needed to make a statistically significant conclusion.

Outcomes data also are very limited. Most notable, Lin et al. (2005), who described the procedure as a single figure of 8 permanent suture through the uterosacral ligament at the level of the ischial spines and through the vaginal cuff, reported a failure rate of $12.8 \%$ (17/133 patients) as defined by anatomic recurrence of apical prolapse $\geq$ Grade 2 BadenWalker with a mean follow-up of 3.2 years. Further analysis of these failures noted that $23.5 \%(4 / 17)$ were associated with prior failed pelvic floor reconstructive surgery. Complications were uncommon at a rate of $2.25 \%$ with no reported ureteral injuries or blood transfusions. The authors substantiate the low estimated blood loss and no ureteral injuries to meticulous hemostasis and ureteral dissection afforded by improved visualization and surgical precision of laparoscopy [24].

Suture complications (exposure, granulation tissue, vaginal discharge, and/or bleeding) recently have gained the attention of surgeons and investigators. Vaginally, Shull described the use of permanent braided sutures extraluminal to the vaginal canal [19]. Subsequent modifications of Shull's technique have incorporated variations on the use of permanent and absorbable sutures for apical support [21, 25-27, 28•]. Kasturi et al. (2011) retrospectively compared apical support outcomes from mostly vaginal uterosacral suspensions using either permanent or delayed-absorbable suture. Results at 1 year did not reveal a significant difference in apical support based on suture selection; however, $22 \%$ (11/50) of the permanent suture group had 
symptomatic suture exposure within 1 year after surgery with 9 of the 11 women ultimately requiring suture removal [29•]. Because this surgical cohort included mostly vaginal cases $\mathrm{n}=110$, (abdominal $\mathrm{n}=2$, laparoscopic $\mathrm{n}=3$ ), the study design was not powered to detect any outcome differences based on surgical approach; that is, the incidence of suture exposures by surgical approach was not determined.

In summary, advocates of uterosacral colpopexy promote the benefits of avoiding inherent risks of mesh-augmented procedures while restoring a more anatomic vaginal axis with high patient satisfaction and moderate durability. In select cases, wherein mesh augmentation may not be preferable to the patient or surgeon, laparoscopic uterosacral ligament colpopexy is a safe and effective native tissue option. On the contrary, in cases where native tissue and pelvic floor muscle integrity is significantly compromised and the degree of prolapse is advanced and/or recurrent from previously failed native tissue repairs, most expert opinion recommends the use of mesh-augmented procedures.

\section{Laparoscopic Sacral Colpopexy/Sacrocolpopexy}

The "gold standard" procedure for apical prolapse remains the abdominal sacral colpopexy that suspends the vaginal vault by reinforcing the anterior and posterior vaginal fibromuscularis with mesh secured to the anterior longitudinal sacral ligament. This procedure has evolved from mesh attachment to just the apex to now extension down the anterior and posterior walls. A comprehensive review reported long-term durability with success rates ranging from 78-100 \% [30]. Nezhat et al. (1994) reported one of the first case series on 15 women who underwent laparoscopic sacral colpopexy with $100 \%$ cure rates for apical prolapse out to 40 months follow-up [3].

Clinical outcomes and comparative data on laparoscopic sacral colpopexy are largely limited to retrospective data. Ganatra et al. (2009) recently reviewed the status of laparoscopic sacrocolpopexy and reported 11 retrospective series with 1,197 total patients over 54.3 study years that demonstrate an overall $94.4 \%$ subjective satisfaction rate, $6.2 \%$ prolapse reoperation rate, and a $2.7 \%$ mesh exposure rate with a mean follow-up of 24.6 months (range 11.4-66 months). Furthermore, conversion rates, operative times, and complications have decreased with increased laparoscopy experience over the past 20 years [31]. Although prospective, randomized, control trials with long-term follow-up are still lacking, one recent U.K. multicenter prospective trial by Freeman et al. (2013) continues to support minimally invasive laparoscopic sacral colpopexy as a clinically equivalent approach to that of the traditional open procedure [4••].

Two prospective, randomized studies compare conventional laparoscopic sacral colpopexy to other techniques. Paraiso et al. (2011) compared conventional laparoscopic $(n=38)$ and robotic $(n=40)$ sacrocolpopexy for vaginal apex prolapse and concluded that robotic-assisted resulted in longer operating times, increased pain, and cost [32••]. Maher et al. (2011) compared laparoscopic sacral colpopexy (LSC) and total vaginal mesh $\left(\mathrm{TVM}^{\circledR}\right)$ for vaginal vault prolapse and concluded at 2 years that LSC had higher satisfaction rates and objective success rates with lower perioperative morbidity and reoperation rates and costs [33••, $34 \bullet]$.

Major complications associated with laparoscopic sacrocolpopexy are uncommon and include rectal injury, small-bowel obstruction, bladder injury, vascular injury, and mesh complications [35, 36]. Ross and Preston (2005) reported 5 -year outcome data on 43 of 51 patients ( $84 \%$ follow-up rate). Two patients had partial small-bowel obstruction secondary to bowel adherence to the mesh and four had mesh exposures at the vaginal apex. Recurrent vaginal prolapse as defined by symptomatic Baden Walker Grade 2 prolapse or any prolapse $\geq$ Grade 3 occurred in three patients (objective cure rate $93 \%$ ) [36]. Warner et al. (2012) retrospectively reviewed 390 cases for complications and quantified the risks of intraoperative and postoperative gastrointestinal complications associated with laparoscopic sacrocolpopexy. The combined rate for ileus and small-bowel obstruction was $1.0 \%$. The bowel injury rate was $1.3 \%$. Prior abdominal surgery was associated with functional GI complications (ileus, SBO, nausea/emesis, $p=0.048)$ but not with bowel injury $(p=0.71)$. The total reoperation rate for SBO or bowel injury remains low at $0.8 \%$ [37•].

Mesh exposure rates associated with laparoscopic sacral colpopexy have been generally lower than the previously reported abdominal data. The reasons are not entirely clear but may involve advances in mesh and suture technology. The cumulative overall mesh exposure rate reported for the abdominal approach was $3.4 \%(70 / 2,178)$ with polypropylene demonstrating the lowest synthetic mesh exposure rate of $0.5 \%(1 / 211)$ [30]. Agarwala et al. (2007) reported zero exposures and recurrences ( $\geq$ Stage II) with exclusive use of polypropylene Gynemesh (Ethicon, Inc., Johnson \& Johnson, Somerville, NJ) in all 72 laparoscopic cases (49 sacral colpopexy, 22 cervicopexy, 1 uteropexy) with $97 \%(70 / 72)$ follow-up rate at 1 year and $66 \%(48 / 72)$ at 2 years [38]. Stepanian et al. (2008) also looked at mesh exposure rates associated with use of macroporous polypropylene mesh. In this cohort of 402 patients who underwent a laparoscopic sacral colpopexy with and without concurrent hysterectomy, authors concluded a low mesh exposure rate of $1.2 \%$ without differences in regards to concurrent laparoscopic-assisted hysterectomy [39]. Experts therefore recommend use of a Type I macroporous monofilament synthetic polypropylene mesh based on relatively low complications [30].

Limited data on modifiable risk factors for mesh exposure, such as concurrent hysterectomy, smoking, and use of xenogenic barriers or biografts, are available. Currently, there is conflicting data on concurrent hysterectomy as a modifiable 
risk factor for mesh exposure. Cundiff et al. (2008) reported an increased associated risk (odds ratio (OR) 4.9), contrary to findings by Nosti et al. (2009) who reported no significant risk (OR 0.95, confidence interval (CI) 0.41-2.18; $p=0.899$ ) [40, 41]. Two studies report smoking as a significant modifiable risk factor (OR 4.4-5.2) [40, 42].

With regards to biografts, two recent studies are notable. The first is a retrospective study that evaluated use of a biograft interlay for abdominovaginal laparoscopic sacral colpoperineopexy technique that noted mesh exposure rates approaching significance with this technique. McDermott et al. (2011) retrospectively reported 12-month outcomes on laparoscopic sacral colpoperineopexies performed either completely abdominally (A-LSCP) or abdominovaginally (AV-LSCP) in cases of vault prolapse with excessive perineal mobility. The posterior combined graft used for the AVLSCP approach included a biograft (Pelvicol ${ }^{\circledR}$, Bard, Murray Hill, NJ) interposed between the polypropylene mesh and vagina in an attempt to protect the vaginal/perineal incision from mesh exposure. The total foreign body reaction rate totaling all mesh exposures, suture exposures, and granulation tissue approached significance $(p=0.06)$ with a rate of $0 \%$ in the A-LSCP group compared with $20 \%$ in the AVLSCP group [43 ${ }^{\bullet}$. A current trial is in progress that will provide 2-year follow-up data on patients randomized to AV-LSCP with or without a biograft overlay that will hopefully provide more conclusive data.

The second study was a prospective, randomized, clinical trial. Culligan et al. (2013) that randomized patients scheduled for laparoscopic sacrocolpopexy either to porcine dermis $(\mathrm{n}=57)$ or polypropylene mesh $(\mathrm{n}=58)$. There were no preoperative differences between groups. This double-blinded, randomized, controlled trial demonstrated similar 12-month objective anatomic cure rates as defined by no prolapse $\mathrm{POPQ} \geq$ Stage II at any postoperative interval $(80.7 \%$ porcine vs. $86.2 \%$ mesh, $p=0.24)$. The "clinical cure" (POP-Q point $\mathrm{C} \leq-5)$ rates also were similar ( $84.2 \%$ porcine vs. $89.7 \%$ mesh, $p=0.96$ ) with significant score improvements in both groups for PFDI-20 and PFIQ-7. No major operative complications were reported in either group [44•].

In summary of current available literature, the laparoscopic approach to sacral colpopexy is a safe and durable procedure to correct apical and complex vault prolapse with reduced perioperative morbidity compared with the open approach when executed by skilled surgeons. Use of Type I macroporous polypropylene mesh is recommended. Utility, benefits, and durability of biografts for sacral colpopexy remain primarily investigational (Table 1).

\section{Concomitant Continence Procedure}

The landmark CARE trial (Colpopexy and Urinary Reduction Efforts) concluded that continent women who undergo abdominal sacrocolpopexy for prolapse significantly benefit from a concurrent Burch colposuspension to reduce postoperative de novo symptoms of stress incontinence [45, 46]. Two recent retrospective, descriptive studies reported outcomes of sacrocolpopexy performed laparoscopically with regard to postoperative de novo stress incontinence. Leruth et al. (2013) reported the incidence of de novo SUI after laparoscopic sacrocolpopexy on a retrospective cohort of 55 women with negative preoperative prolapse reduction stress testing (absence of leakage with cough test and reduction urodynamics). At a mean follow-up of 25 months (range 12$48)$, more than half $(54.5 \%, 30 / 55)$ reported subjective de novo SUI, $13(23.6 \%)$ had a positive cough test, and 9 $(16.4 \%)$ underwent an interval sling procedure. Although the univariate analyses suggested an association between preoperative subjective SUI and Stage 3-4 cystocele with higher risks of both postoperative subjective and objective de novo SUI, the multivariate analyses only identified preoperative subjective SUI as the sole independent predictor of postoperative subjective de novo SUI (relative risk (RR) 4.03; $95 \%$ CI 1.16-14.09), objective de novo SUI (RR 4.67; $95 \%$ CI 1.14-19.23), and subsequent anti-SUI surgery (RR 6.17; $95 \%$ CI 1.3-29.41) [47••]. Park et al. (2012) retrospectively reviewed the risk of a second surgery in women who underwent preoperative reduction stress testing (RST) before laparoscopic sacral colpopexy for symptomatic prolapse. Women with positive RST had a concomitant midurethral sling procedure and those with negative RST did not. In total, $18.6 \%$ of continent women with negative preoperative RST who did not get a sling at the time of laparoscopic sacral colpopexy had a second surgery for de novo urodynamic stress incontinence; $7.3 \%$ of women with positive RST and concomitant midurethral slings underwent sling revision. The total incidence of a second surgery for either sling placement or sling revision was $13 \%(19 / 152)$. [48•].

In summary, the incidence of symptomatic postoperative de novo SUI after sacrocolpopexy is significant, regardless of route (open or laparoscopic). Similar to the CARE trial open approach outcomes, women undergoing sacral colpopexy by laparoscopic approach are at risk for postoperative de novo SUI and, therefore, will likely benefit from a concurrent continence procedure. Furthermore, despite negative preoperative reduction testing, women may have a $16-18 \%$ risk of requiring an interval sling after a laparoscopic sacral colpopexy [47••, 48•].

\section{Laparoscopic Hysteropexy (Uterosacral Hysteropexy,}

Sacrohysteropexy or Sacrocervicopexy)

Few descriptive and observational studies report variations of sacral colpopexy and uterosacral ligament suspension as a means to correct apical prolapse without hysterectomy for women who desire uterine preservation. Rosenblatt et al. (2008) evaluated apical support in a retrospective case series 
Table 1 Comparison studies on sacral colpopexy

\begin{tabular}{|c|c|c|}
\hline Authors & Comparisons & Outcomes \\
\hline Freeman et al.* (IUJ 2013) & ASC vs. LSC & $\begin{array}{l}\text { POP-Q points C were similar at } 1 \text { year. } \\
\text { ASC }-6.63 \mathrm{~cm} \text { vs. LSC }-6.67 \mathrm{~cm} \\
\text { Subjective outcomes were similar at } 1 \text { year. } \\
\text { ASC } 90 \% \text { vs. LSC } 80 \% \text { were "much better" at } 1 \text { year. } \\
\text { Difference between means at } 1 \text { year }=0.026 \mathrm{~cm}(\mathrm{CI}-0.676 \text { to } 0.747) \\
\quad \text { confirming noninferiority. }\end{array}$ \\
\hline Siddiqui et al. (AJOG 2012) & ASC vs. RSC & $\begin{array}{l}\text { Similar surgical failure rates based on composite outcomes } \\
\quad \text { (recurrent symptoms and/or repeat surgery) at } 1 \text { year. } \\
\text { RSC } 8 \%(7 / 86) \text { vs. ASC } 4 \%(12 / 304) ; p=0.16 \text {. } \\
\text { Similar anatomic failure rates at } 1 \text { year. } \\
\text { RSC } 6 \%(4 / 70) \text { vs. } 6 \%(16 / 289) ; p=0.57 .\end{array}$ \\
\hline Paraiso et al. (Obstet Gynecol 2011)* & LSC vs. RSC & $\begin{array}{l}\text { No differences in anatomical or functional outcomes at } 1 \text { year. } \\
\text { RSC longer anesthesia time, operating time, sacrocolpopexy time, } \\
\text { total suturing time. } \\
\text { RSC more postoperative pain at rest and with activity, } \\
\quad p<0.005 \text {. } \\
\text { RSC higher costs, } p=0.008 \text {. }\end{array}$ \\
\hline Pasic et al. (JMIG 2010) & LSC vs. RSC & $\begin{array}{l}\text { RSC high cost in both outpatient and inpatient settings. } \\
\text { RSC surgical times longer } 3.2 \mathrm{hr} \text { vs. } 2.8 \mathrm{hr} \text { (CI 3.21-3.23). }\end{array}$ \\
\hline Judd et al. (JMIG 2010) & LSC vs. RSC vs. ASC & RSC higher costs than either ASC or LSC. \\
\hline Maher et al. (AJOG 2012) & LSC vs. TVM & $\begin{array}{l}\text { LSC lower mean primary clinical and total economic cost. } \\
\text { LSC higher labor costs offset by TVM consumable inpatient, } \\
\text { opportunity, reoperation costs. }\end{array}$ \\
\hline Maher et al.** (AJOG 2011) & LSC vs. TVM & $\begin{array}{l}\text { LSC longer operative time, reduced inpatient days, } \\
\text { quicker return to activity. } \\
\text { Total objective success rates at all sites: } \\
\text { LSC } 77 \%(41 / 53) \text { vs. TVM } 43 \%(23 / 55), p<0.001 \text {. } \\
\text { Reoperation rate: } \\
\text { LSC } 5 \%(3 / 53) \text { vs. TVM } 22 \%(12 / 55), p=0.006 \text {. }\end{array}$ \\
\hline McDermott et al. (IUJ 2011) & A-LSCP vs. AV-LSCP & $\begin{array}{l}\text { Results at } 6-12 \text { months postoperative: } \\
\text { No differences of POP-Q or stage of prolapse, } p>0.05 \\
\text { No difference in surgical satisfaction, } p=0.8 \\
\text { A-LSCP lower rates of mesh exposure and dyspareunia, } p>0.05 \\
\text { AV-LSCP fewer prolapse symptoms, } p=0.01 \text {. }\end{array}$ \\
\hline
\end{tabular}

*Randomized controlled trial. **Randomized trial. ASC, abdominal open sacrocolpopexy; LSC, laparoscopic sacral colpopexy; RSC, robotic sacral colpopexy; TVM, Total Vaginal Mesh; A-LSCP, abdominal-laparoscopic sacral colpopexy; AVLSCP, abdomino-vaginal laparoscopic sacral colpopexy

of 40 women who underwent laparoscopic sacrocervicopexy where synthetic mesh was used to attach the "distal uterosacral ligaments and posterior endopelvic fascia to the anterior longitudinal ligament of the sacral promontory." Mean POP-Q C points were followed from -1.13 preoperatively to $-5.26(-3$ to -8$)$ postoperatively at 6 months and to $-4.84(-3$ to -7$)$ at 1 year postoperatively [49]. Price et al. (2010) prospectively evaluated 51 women $-80 \%$ with preoperative Baden-Walker grade 3 uterine prolapse-who underwent a laparoscopic hysteropexy technique using a bifurcated polypropylene mesh wrapped around the anterior cervix through bilateral broad ligament windows and fixated to the sacral promontory. Shortterm 10-week to 6-month postoperative subjective and objective outcomes reported one failure (symptomatic Baden-Walker Grade 2) and no major intraoperative complications. Significant subjective improvements in prolapse symptoms, sexual wellbeing, and quality of life were observed as evaluated by the International Consultation on Incontinence Questionnaire for Vaginal Symptoms (ICIQ-VS) [50•]. Bedford et al. (2013) described laparoscopic uterosacral suspension with and without uterine preservation. This large retrospective cohort from 1999-2010 included 104 laparoscopic uterosacral hysteropexy and 160 [laparovaginal] hysterectomies with laparoscopic uterosacral colpopexy. With a median follow-up of 2.5 years, the total objective failure rate (POPQ Stage $\geq 2$ in all compartments) for hysteropexy was $52.9 \%$ and for hysterectomy with 
uterosacral colpopexy was $37.5 \%(p=0.02)$. Repeat overall operation rates were similar (hysteropexy $27.9 \%$ vs. hysterectomy/uterosacral colpopexy $20.6 \%, p=0.23$ ). Specific apical failures were $24 \%$ for hysteropexy and $13.1 \%$ for hysterectomy/uterosacral colpopexy $(p<0.03)[51 \bullet]$. Authors support laparoscopic hysteropexy as a reasonable alternative to hysterectomy with uterosacral colpopexy given the similar reoperations rates.

In summary, data are limited on long-term durability of hysteropexy procedures. Therefore, patients interested in uterine preservation for the sole reason of desiring future fertility must be aware of the probable deleterious effects that pregnancy may have on the durability of reconstructive procedures. Furthermore, while access to uterine and cervical surveillance and possible need for hysterectomy for future pathology may be more difficult after pelvic floor reconstruction, recommendations to continue surveillance should be continued and emphasized.

\section{Robotics in Pelvic Reconstructive Surgery}

Shariati reported the first case series of 77 women who underwent da Vinci ${ }^{\circledR}$ assisted laparoscopic sacrocolpopexy from 2003 to 2005 with high patient satisfaction and success rates out to 1 year postsurgery [52]. In 2005, the FDA approved the da Vinci ${ }^{\circledR}$ Surgical System (Intuitive Surgical, Inc. Sunnyvale, CA) for use in gynecologic surgery. Advocates of roboticassisted laparoscopy have since postulated the various advantages over conventional laparoscopy in regards to motion mechanics and 3-D visualization. The more ergonomic features are thought to enhance surgeon dexterity via the telerobotic platform that utilizes specialized endo-wrist instruments that provide the surgeon with the same 6-degrees of flexibility as the human wrist while minimizing physiologic tremors and large movements. Nezhat et al. (2006) in his case series of 15 patients undergoing various gynecologic procedures by both conventional and robotic laparoscopy highlighted potential advantages of high-definition, three-dimensional video in regards to enhancing surgical precision and depth perception. Notable disadvantages of robotics also were noted, such as steep learning curve, longer operative times due to assembly and disassembly, lack of tactile haptics, significant equipment and hospital costs, and lack of universal availability in hospitals $[9 \bullet, 32 \bullet, 53]$.

Recent data on robotic sacral colpopexy have demonstrated equivalent advantages over the open procedure as previously demonstrated by conventional laparoscopy in terms of less blood loss, shorter hospitalizations, and less pain while maintaining successful anatomical outcomes with relatively low complication rates [54•]. Siddiqui et al. (2012) compared 1 -year outcomes after robotic and abdominal sacrocolpopexy on a large retrospective cohort of 447 women (125 robotic vs. 322 abdominal). They concluded robotics provides an alternative approach to open sacrocolpopexy with similar symptomatic and anatomic success as compared to the gold-standard abdominal approach with no statistically significant differences in surgical failures when analyzed as a composite outcome (RSC 7/86 [8\%] vs. ASC 12/304 [4\%]; $p=0.16$ ) and also when anatomic failures were considered (RSC 4/70 [6\%] vs. ASC 16/289 [6\%]; $p=0.57$ ) [55•]. Geller et al. (2012) recently published longterm clinical outcome data with a mean follow-up of 44 months on a retrospective contemporary cohort of 51 women (23 robotic, 28 abdominal) who underwent either robotic or abdominal sacrocolpopexy between March 2006 and October 2007. Postoperative POP-Q improvements were similar between routes $(\mathrm{C}-8$ vs. $-7, \mathrm{Aa}-2.5$ vs. $-2.3, \mathrm{Ap}-2$ vs. -2 , $p>0.05$ ). Improvements in pelvic floor functions as measured by validated questionnaires PFDI-20, PFIQ-7, and PISQ-12 were similar between routes $(p>0.05)$. Mesh exposures also were similar ( $8 \%$ robotic vs. $7 \%$ abdominal) [54•]. The authors conclude robotic sacrocolpopexy is an equivalently effective and durable alternative to the open procedure based on several published 1-year studies and now 3-year data [54•, 55 . Similar equivalence has been demonstrated between robotic and conventional laparoscopic sacral colpopexy in regards to short-term outcomes of improved pelvic support and satisfied functional outcomes at 1-year after surgery [32••]. A randomized, comparative, effectiveness trial, ACCESS (Abdominal Colpopexy: Comparison of Endoscopic Surgical Strategies) that will look primary at costs, is currently in progress at UCLA (Los Angeles) and Loyola (Chicago, IL) [56•].

In summary, robotic-assisted pelvic surgery has gained widespread acceptance due to improved efficiency of skilled surgeons over time and studies that continue to support comparable safety and efficacy compared with both open approach and conventional laparoscopy. However, there remains an unclear benefit over conventional laparoscopy, especially for most skilled laparoscopic surgeons who perform both approaches well [32••].

\section{Laparoendoscopic Single-Site Surgery (Less) for Pelvic Prolapse}

The use of LESS surgical technique for prolapse surgeries is limited to few expert surgeon case reports. Future studies are needed to determine whether the LESS approach provides any benefit over conventional or robotic-assisted laparoscopic approaches $[57,58,59 \bullet]$.

Posterior Compartment

Laparoscopic Rectocele Repair (Colpoperineopexy, Abdominovaginal Rectocele Repair)

Similar to paravaginal repairs, data on laparoscopic rectocele repairs, as an isolated procedure, are sparse. Lyons described 
one of the first laparoscopic rectocele repairs using mesh in 1997 [60]. Current versions of laparoscopic rectocele repairs derive its origins from the abdominal technique described by Cundiff et al. (1997) that extends the abdominal sacral colpopexy posterior mesh down to the rectovaginal septum and perineal body, thereby correcting posterior defects and providing perineal support [61]. Link et al. (2004) subsequently adopted this sacral colpopexy variation for laparoscopy [62]. Currently, multiple modifications exist to accommodate the variable extent of posterior and perineal defects that are unique to each prolapse. McDermott et al. (2011) describes the Abdomino-Vaginal Laparoscopic Sacral Colpoperineopexy approach (AV-LSCP) that utilizes a biograft interlay between the polypropylene mesh and the posterior vaginal muscularis. It is used in cases where there is excessive perineal mobility. The biograft portion is attached to the rectovaginal septum through the posterior vaginal dissection that allows for the wide and distal attachment of the polypropylene portion of the graft to the levator fasciae at the level of the ischial spines. This posterior combined graft is then brought into the peritoneal cavity through the enterocele sac and the remainder of the sacral colpopexy is completed laparoscopically. This technique aims to effectively obliterate the levator hiatus thereby preventing and treating rectoceles, enteroceles and apical posterior defects. McDermott reported 12-month outcomes on this AV-LSCP technique compared to the laparoscopic only sacral colpopexy (A-LSCP) and noted no significant differences in anatomical posterior POP-Q outcomes (or any other POP-Q points) up to 1 year after surgery. Additionally, there were no reoperations for recurrent prolapse in this surgical cohort. Notably, the AV-LSCP group demonstrated significantly lower recurrent prolapse symptoms (25\% A-LSCP vs. $2 \%$ AV-LSCP, $p=0.01$ ) [43•].

In summary, laparoscopic rectocele repairs, as an isolated procedure, are uncommon. Therefore, current literature lacks data on surgical technique and clinical outcomes to consider this approach a viable alternative to the conventional posterior colporrhaphy. Most experts continue to support the vaginal native tissue posterior repair approach due to its relatively high objective and subjective cure rates [63].

\section{Laparoscopic Enterocele Repair (Culdoplasty)}

Current literature on laparoscopic enterocele repairs remains descriptive only. Laparoscopic enterocele repair surgical principles are identical to the open and vaginal procedures starting with identifying the enterocele sac, excising the redundant peritoneum, and reapproximating the rectovaginal and pubocervical fibromuscular tissue in the midline to repair the defect and obliterate the cul-de-sac. Enterocele repairs often are performed concomitantly with apical suspensions as previously described in the uterosacral ligament suspension and sacral colpopexy/perineopexy sections [19, 26, 43•, 64].

\section{Conclusions}

The notion of minimally invasive reconstructive surgery continues to evolve as advancing biomedical technology not only facilitates but also challenges previously established surgical techniques. Well-designed, prospective, randomized, controlled trials are needed if we are to shift the paradigm of previously established "gold standards" that have demonstrated proven efficacy and durability over time. Until then, the experienced surgeon must continue to individualize risks, benefits and alternatives of various skill-sets in order to optimize the patients' overall surgical outcome.

The most important tenet to adopt when learning minimally invasive surgical techniques is to preserve the surgical principles of previously established "gold-standard" conventional techniques. The age of laparoscopy is currently in an unusual phase between being "advanced" (compared with vaginal and open approaches) and "conventional" (compared with robotics). Nonetheless, laparoscopy is a means to provide minimal access surgery. When executed by experienced surgeons, laparoscopic pelvic reconstructive surgery leads the way in reducing perioperative morbidity while preserving efficacy and durability of pelvic organ prolapse treatment.

\section{Compliance with Ethics Guidelines}

Conflict of Interest Michelle M. Takase-Sanchez declares that she has no conflict of interest.

Douglass S. Hale declares that he has no conflict of interest.

Human and Animal Rights and Informed Consent This article does not contain any studies with human or animal subjects performed by any of the authors.

\section{References}

Papers of particular interest, published recently, have been highlighted as:

- Of importance

•. Of major importance

1. Diwan A, Rardin CR, Strohsnitter WC, et al. Laparoscopic uterosacral ligament uterine suspension compared with vaginal hysterectomy with vaginal vault suspension for uterovaginal prolapse. Int Urogynecol J Pelvic Floor Dysfunct. 2006;17:7983.

2. Medina C, Takacs P. Laparoscopic uterosacral uterine suspension: a minimally invasive technique for treating pelvic organ prolapse. J Minim Invasive Gynecol. 2006;13(5):472-5.

3. Nezhat $\mathrm{CH}$, Nezhat F, Nezhat C. Laparoscopic sacral colpopexy for vaginal vault prolapse. Obstet Gynecol. 1994;84:885-8.

4. •- Freeman RM, Pantazis K, Thomson A, et al. A randomized controlled trial of abdominal versus laparoscopic sacrocolpopexy for the treatment of post hysterectomy vaginal vault prolapse: LAS study. Int Urogynecol J. 2013;24(3):377-84. This study noted 
similar objective and subjective outcomes between the abdominal and laparoscopic approaches.

5. Paraiso MF, Walters MD, Rackley RR, et al. Laparoscopic and abdominal sacral colpopexies: a comparative cohort study. Am J Obstet Gynecol. 2005;192:1752-8.

6. Hsiao KC, Latchamsetty K, Govier FE, et al. Comparison of laparoscopic and abdominal sacrocolpopexy for the treatment of vaginal vault prolapse. J Endourol. 2007;21:926-30.

7. Klauschie JL, Suozzi BA, O'Brien MM, et al. A comparison of laparoscopic and abdominal sacral colpopexy: objective outcome and perioperative differences. Int Urogynecol $\mathrm{J}$ Pelvic Floor Dysfunct. 2009;20:273-9.

8. Saidi MH, Vancaillie TG, White AJ, et al. Complications of major operative laparoscopy. A review of 452 cases. J Reprod Med. 1996;41(7):471-6.

9. • Pasic RP, Rizzo JA, Fang H, et al. Comparing robot-assisted with conventional laparoscopic hysterectomy: impact on cost and clinical outcomes. J Minim Invasive Gynecol. 2010;17(6):730-8. This study showed higher costs and surgical times for robotic-assisted compared to conventional laparoscopy.

10. • Judd JP, Siddiqui NY, Barnett JC, et al. Cost-minimization analysis of robotic- assisted, laparoscopic, and abdominal sacrocolpopexy. J Minim Invasive Gynecol. 2010;17(4):493-9. This study showed robotics was most expensive. Robotic and laparoscopic became cost equivalent when robotic surgical time was decreased.

11. White GR. Cystocele - a radical cure by suturing lateral sulci of the vagina to the white line of pelvic fascia. 1909.

12. Delancey JO. Fascial and muscular abnormalities in women with urethral hypermobility and anterior vaginal wall prolapse. Am J Obstet Gynecol. 2002;187:93-8.

13. Summers A, Winkel LA, Hussain HK, DeLancey JO. The relationship between anterior and apical compartment support. Am J Obstet Gynecol. 2006;194(5):1438-43.

14. Rooney K, Kenton K, Mueller ER, et al. Advanced anterior vaginal wall prolapse is highly correlated with apical prolapse. Am J Obstet Gynecol. 2006;195(6):1837-40.

15. Chen L, Ashton-Miller JA, Hsu Y, DeLancey JO. Interaction among apical support, levator ani impairment, and anterior vaginal wall prolapse. Obstet Gynecol. 2006;108:324-32.

16. Behnia-Willison F, Seman EI, Cook JR, et al. Laparoscopic paravaginal repair of anterior compartment prolapse. J Minim Invasive Gynecol. 2007;14:475-80.

17. - Shippey SH, Quiroz LH, Sanses TVD, et al. Anatomic outcomes of abdominal sacrocolpopexy with or without paravaginal repair. Int Urogynecol J. 2010;21:279-83. This study showed a trend toward improved anterior prolapse repair outcomes with concurrent paravaginal repairs.

18. Maher C, Baessler K. Surgical management of anterior vaginal wall prolapse: an evidence-based literature review. Int Urogynecol J. 2006;17:195-201.

19. Shull BL, Bachofen C, Coates KW, Kuehl TJ. A transvaginal approach to repair of apical and other associated sites of pelvic organ prolapse with uterosacral ligaments. Am J Obstet Gynecol. 2000;183:1365-73.

20. - Margulies RU, Rogers MA, Morgan DM. Outcomes of transvaginal uterosacral ligament suspension: systematic review and metaanalysis. Am J Obstet Gynecol. 2010;202:124-34. This systematic review provides a metanalysis of anatomic outcomes and a summary of subjective outcomes. Pooled rates of success for anterior, posterior and apical outcomes were $81.2 \%$.

21. Barber MD, Visco AG, Weidner AC, et al. Bilateral uterosacral vaginal vault suspension with site-specific endopelvic fascia defect repair for treatment of pelvic organ prolapse. Am J Obstet Gynecol. 2000;183:1402-10.

22. Culligan PJ, Miklos JR, Murphy M, et al. The tensile strength of uterosacral ligament sutures: a comparison of vaginal and laparoscopic techniques. Obstet Gynecol. 2003;101:500-3.
23. Rardin CR, Erekson EA, Sung VW, et al. Uterosacral colpopexy at the time of vaginal hysterectomy: comparison of laparoscopic and vaginal approaches. J Repod Med. 2009;54(5):273-80.

24. Lin LL, Phelps JY, Liu CY. Laparoscopic vaginal vault suspension using uterosacral ligaments: a review of 133 cases. J Minim Invasive Gynecol. 2005;12:216-20.

25. Silva WA, Pauls RN, Segal JL, et al. Uterosacral ligament vault suspension: five-year outcomes. Obstet Gynecol. 2006;108(2):25563.

26. Karram M, Goldwasser S, Kleeman S, et al. High uterosacral vaginal vault suspension with fascial reconstruction for vaginal repair of enterocele and vaginal vault prolapse. Am J Obstet Gynecol. 2001;185(6):1339-42.

27. • Wong MJ, Rezvan A, Bhatia NN, Yazday T. Uterosacral ligament vaginal vault suspension using delayed absorbable monofilament suture. Int Urogynecol J. 2011;22(11):1389-94. This study showed $3.5 \%$ suture complications with polyglyconate absorbable suture as compared to the $44.6 \%$ rate of suture erosion with permanent suture.

28. • Yazdany T, Yip S, Bhatia NN, Nguyen JN. Suture complications in a teaching institution among patients undergoing uterosacral ligament suspension with permanent braided suture. Int Urogynecol J. 2010;21(7):813-8. This study showed that permanent polyester braided suture had $44.6 \%$ suture complications.

29. - Kasturi S, Bentley-Taylor M, Woodman PJ, et al. High uterosacral ligament vaginal vault suspension: comparison of absorbable vs permanent suture for apical fixation. Int Urogynecol J. 2012;23:941-5. This study showed permanent suture erosion rate of $22 \%$ and no difference in apical support when compared to absorbable suture in mostly vaginal approach uterosacral suspensions.

30. Nygaard IE, McCreery R, Brubaker L, et al. Pelvic floor disorders network. Abdominal sacrocolpopexy: a comprehensive review. Obstet Gynecol. 2004;104:805-23.

31. Ganatra AM, Rozet F, Sanchez-Salas R, et al. The current status of laparoscopic sacrocolpopexy: a review. Eur Urol. 2009;55(5):1089103.

32. •- Paraiso MF, Jelovsek JE, Frick A, et al. Laparoscopic compared with robotic sacrocolpopexy for vaginal prolapse: a randomized controlled trial. Obstet Gynecol. 2011;118(5):1005-13. This study showed robotics approach was associated with longer operative times, pain and cost than conventional laparoscopy.

33. • Maher CF, Feiner B, DeCuyper EM, et al. Laparoscopic sacral colpopexy versus total vaginal mesh for vaginal vault prolapse: a randomized trial. Am J Obstet Gynecol. 2011;204:360.e1-7. This study showed laparoscopic had a higher satisfaction rate and objective success rate than TVM with lower perioperative morbidity and reoperation rate.

34. - Maher CF, Connelly LB. Cost minimization analysis of laparoscopic sacral colpopexy and total vaginal mesh. Am J Obstet Gynecol. 2012;206:433.e1-7. This study showed laparoscopic has lower costs than TVM.

35. Cosson M, Rajabally R, Bogaert E, et al. Laparoscopic sacrocolpopexy, hysterectomy, and Burch colposuspension: feasibility and short-term complications of 77 procedures. JSLS. 2002;6:115-9.

36. Ross JW, Preston M. Laparoscopic sacrocolpopexy for severe vaginal vault prolapse: five-year outcome. J Minim Invasive Gynecol. 2005;12(3):221-6.

37. - Warner WB, Vora S, Alonge A, et al. Intraoperative and postoperative gastrointestinal complications associated with laparoscopic sacrocolpopexy. Female Pelvic Med Reconstr Surg. 2012;18(6):321-4. This study confirmed that GI complications remain low with laparoscopic sacrocolpopexy and that previous abdominal surgery is associated with higher risk of postoperative functional GI complications but not bowel injury. 
38. Agarwala N, Hasiak N, Shade M. Laparoscopic sacral colpopexy with Gynemesh as graft material - experience and results. J Minim Invasive Gynecol. 2007;14:577-83.

39. Stepanian SG, Miklos JR, Moore RD, Mattox TF. Risk of mesh extrusion and other mesh-related complications after laparoscopic sacral colpopexy with or without concurrent laparoscopic-assisted vaginal hysterectomy: experience of 402 patients. J Minim Invasive Gynecol. 2008;15:188-96.

40. Cundiff GW, Varner E, Visco AG, et al. Risk factors for mesh/ suture erosion following sacral colpopexy. Am J Obstet Gynecol. 2008;199:688.e1-5.

41. Nosti PA, Lowman JK, Zollinger TW, et al. Risk of mesh erosion after abdominal sacral colpoperineopexy with concomitant hysterectomy. Am J Obstet Gynecol. 2009;201(5):541.e1-4.

42. Lowman JK, Woodman PJ, Nosti PA, et al. Tobacco use is a risk factor for mesh erosion after abdominal sacral colpoperineopexy. Am J Obstet Gynecol. 2008;198(5):561.e1-4.

43. - McDermott CD, Park J, Terry CL, et al. Laparoscopic sacral colpoperineopexy: abdominal versus abdominal-vaginal posterior graft attachment. Int Urogynecol J. 2011;22(4):469-75. This study showed comparable anatomic outcomes with differing mesh erosion rates and subjective outcomes.

44. - Culligan PJ, Salamon C, Priestley JL, Shariati A. Porcine dermis compared with polypropylene Mesh for Laparoscopic Sacrocolpopexy. Obstet Gynecol. 2013;121(1):143-51. This study showed similar subjective and objective outcomes at 12 months.

45. Brubaker L, Cundiff GW, Fine P, et al. Pelvic floor disorders network. Abdominal sacrocolpopexy with Burch colposuspension to reduce urinary stress incontinence. N Engl J Med. 2006;354:155766.

46. Brubaker L, Nygaard I, Richter HE, et al. Two-year outcomes after sacrocolpopexy with and without Burch to prevent stress urinary incontinence. Obstet Gynecol. 2008;112:49-55.

47. • Leruth J, Fillet M, Waltregny D. Incidence and risk factors of postoperative stress urinary incontinence following laparoscopic sacrocolpopexy in patients with negative preoperative prolapse reduction stress testing. Int Urogynecol J. 2013;24(3):485-91. This study showed despite negative preoperative reduction testing, $54.5 \%$ had subjective SUI postoperative with 1 in 6 eventually having a sling surgery by 1 year postop.

48. - Park J, McDermott CD, Terry CL, Bump RC, Woodman PJ, Hale DS. Use of preoperative prolapse reduction stress testing and the risk of a second surgery for urinary symptoms following laparoscopic sacral colpoperineopexy. Int Urogynecol J. 2012;23(7):85764. This study showed that the use of preoperative reduction stress testing reduced the risk of a second surgery.

49. Rosenblatt PL, Chelmow D, Ferzandi TR. Laparoscopic sacrocervicopexy for the treatment of uterine prolapse: a retrospective case series report. J Minim Invasive Gynecol. 2008;15(3):26872

50. Price N, Slack A, Jackson S. Laparoscopic hysteropexy: the initial results of a uterine suspension procedure for uterovaginal prolapse. BJOG. 2010;117:62-8. This study supports laparoscopic hysteropexy as a feasible and effective uterine preservation prolapse surgery.
51. • Bedford ND, Seman EI, O'Shea RT, Keirse MJNC. Effect of uterine preservation on outcome of laparoscopic uterosacral suspension. J Minim Invasive Gynecol Jan 2013 Jan;20, In press. This study showed despite superior anatomic outcomes with hysterectomy, repeat operations were not significantly different between laparoscopic uterosacral suspensions with and without hysterectomy.

52. Shariati A, Maceda JS, Hale DS. Da Vinci assisted laparoscopic sacrocolpopexy: surgical technique on a cohort of 77 patients. J pelvic Med Surg. 2008;14:163-71.

53. Nezhat C, Saberi NS, Shahmohamady B, Nezhat F. Roboticassisted laparoscopy in gynecological surgery. J Soc Laparoendosc Surg. 2006;10:317-20.

54. - Geller EJ, Parnell BA, Dunivan GC. Robotic vs. abdominal sacrocolpopexy: 44- month pelvic floor outcomes. Urology. 2012;79:532-6. This study showed similar long-term outcomes.

55. • Siddiqui NY, Geller EJ, Visco AG. Symptomatic and anatomic 1year outcomes after robotic and abdominal sacrocolpopexy. Am J Obstet Gyneol. 2012;206:435.e1-5. This study showed similar anatomic and functional outcomes.

56. - Mueller ER, Kenton K, Tarnay C, et al. Abdominal colpopexy: comparison of endoscopic surgical strategies (ACCESS). Contemp Clin Trials. 2012;33(5):1011-8. This is a multicenter study to primarily assess cost in a comparative effectiveness trial between laparoscopic conventional versus robotic sacrocolpopexy.

57. • Behnia-Willison F, Garg A, Keirse MJ. A Laparoendoscopic singlesite surgery approach to mesh sacrohysteropexy. Case Rep Med. 2013;2013:641675. Epub 2013 Feb 25. This case report describes a LESS technique with posterior attachment of mesh to the sacral promontory and with concurrent anterior and posterior repair with biologic grafts with no objective prolapse at 18 months follow-up.

58. - Marcus-Braun N, von Theobald P. Single port laparoscopic sacrohysteropexy in a young patient presenting with grade III uterine prolapse and rectocele. Int Urogynecol J. 2013 Jan 24 [Epub ahead of print]. This video describes a LESS procedure for Stage III prolapse using the two mesh pieces wrapped around the cervix through the broad ligament.

59. - Ingber MS, Colton MD, Zimmerman GE. Laparoendoscopic single-site (LESS) hysteropexy. Updates Surg. 2012;64(1):53-7. This is a report of LESS procedure done with a mesh to the posterior uterus and attached to the sacral promontory.

60. Lyons TL, Winer WK. Laparoscopic rectocele repair using polyglactin mesh. J Am Assoc Gynecol Laparosc. 1997;4:381-4.

61. Cundiff GW, Harris RL, Coates K, et al. Abdominal sacral colpoperineopexy: a new approach for correction of posterior compartment defects and perineal descent associated with vaginal vault prolapse. Am J Obstet Gynecol. 1997;177(6):1345-53.

62. Link RE, Su LM, Bhayani SB, Wright EJ. Laparoscopic sacral colpoperineopexy for treatment of perineal body descent and vaginal vault prolapse. Urology. 2004;64(1):145-7.

63. Paraiso MF, Barber MD, Muir TW, Walters MD. Rectocele repair: a randomized trial of three surgical techniques including graft augmentation. Am J Obstet Gynecol. 2006;195(6):1762-71.

64. Cook JR, Seman EI, O'Shea RT. Laparoscopic treatment of enterocele: a 3-year evaluation. Aus NZ J Obstet Gynecol. 2004;44:107-10. 